\title{
Early clinical outcome of aortic transcatheter valve-in-valve implantation in the Nordic countries
}

\author{
Leo Ihlberg, MD, PhD, ${ }^{a}$ Henrik Nissen, MD, PhD, ${ }^{b}$ Niels-Erik Nielsen, $\mathrm{MD},{ }^{\mathrm{c}}$ Andreas Rück, MD, ${ }^{\mathrm{d}}$ \\ Rolf Busund, MD, PhD, ${ }^{\mathrm{e}}$ Kaj-Erik Klaarborg, MD ${ }^{\mathrm{f}}$ Lars Soendergaard, MD, PhD, ${ }^{\mathrm{g}}$ Jan Harnek, MD, \\ Heikki Miettinen, MD, PhD, ${ }^{i}$ Markku Eskola, MD, PhD ${ }^{\mathrm{j}}$ Alexander Wahba, $\mathrm{MD}, \mathrm{PhD},{ }^{\mathrm{k}}$ and \\ Mika Laine, MD, $\mathrm{PhD}^{\mathrm{a}}$
}

Objective: Transcatheter valve-in-valve implantation has emerged as an option, in addition to reoperative surgical aortic valve replacement, to treat failed biologic heart valve substitutes. However, the clinical experience with this approach is still limited. We report the comprehensive experience of transcatheter valve-in-valve implantation in the Nordic countries from May 2008 to January 2012.

\begin{abstract}
Methods: A total of 45 transcatheter aortic valve-in-valve implantations were performed during the study period in 11 centers. The mean age of the patients was 80.6 years (range, 61-91), 26 were male and 19 were female, and the mean EuroSCORE, EuroSCORE II, and Society of Thoracic Surgeons score was 35.4, 16.3, and 14.6, respectively. The type of failure was stenosis and combined in 58\% (mean and peak aortic valve gradient, 77 and $45 \mathrm{~mm} \mathrm{Hg}$, respectively) and regurgitation in $42 \%$ of cases. The SAPIEN/XT (Edwards LifeSciences, Irvine, Calif) and CoreValve (Medtronic Inc, Minneapolis, Minn) system was used in 33 and 12 cases, respectively. The access route was transapical in 25 , transfemoral in 17 , transaortic in 2 , and subclavian in 1 case. The mean follow-up was 14.4 months. The periprocedural and postoperative outcomes were assessed using the Valve Academic Research Consortium criteria.
\end{abstract}

Results: No intraprocedural mortality occurred. The technical success rate was $95.6 \%$ ( 1 second valve implantation, 1 conversion to open surgery). The all-cause 30-day mortality was $4.4 \%$ ( 1 cardiac-related and 1 aspiration pneumonia). The major complications within 30 days included stroke in $2.2 \%$, periprocedural myocardial infarction in $4.4 \%$, and major vascular complication in $2.2 \%$ of patients. At 1 month, all but 1 patient had either no or mild paravalvular leakage, with a mean and peak valve gradient of $17 \mathrm{~mm} \mathrm{Hg}$ (range, 4-38) and $30 \mathrm{~mm} \mathrm{Hg}$ (range, 7-68), respectively. The mean gradient was greater than $20 \mathrm{~mm} \mathrm{Hg}$ in $17 \%$ of patients and remained unchanged at 12 months. The 1-year survival was $88.1 \%$.

Conclusions: Transcatheter valve-in-valve implantation is widely performed, albeit in small numbers, in most centers in the Nordic countries. The short-term results were excellent in this high-risk patient population, demonstrating a low incidence of device- or procedure-related complications. However, a considerable number of patients were left with suboptimal systolic valve performance with unknown long-term effects, warranting close surveillance after transcatheter valve-in-valve implantation. (J Thorac Cardiovasc Surg 2013;146:1047-54)

Heart valve replacement with a biologic valve prosthesis is an accepted treatment modality, especially for elderly patients, and a trend has been seen toward choosing bioprosthetic valves over mechanical valves for younger

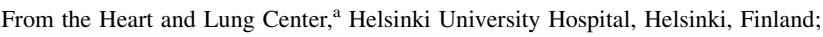
Department of Cardiology, ${ }^{\mathrm{b}}$ Odense University Hospital, Odense, Denmark; Department of Cardiology, ${ }^{\mathrm{c}}$ Linköping University Hospital, Linköping, Sweden; Department of Cardiology, ${ }^{\mathrm{d}}$ Karolinska University Hospital, Stockholm, Sweden; Department of Cardiothoracic and Vascular Surgery, ${ }^{\mathrm{e}}$ University Hospital of North Norway, Troms $\emptyset$, Norway; Department of Cardiothoracic Surgery, ${ }^{f}$ Aarhus University Hospital, Aarhus, Denmark; Department of Cardiology, ${ }^{g}$ Rigshospitalet, Copenhagen, Denmark; Department of Cardiology, ${ }^{\text {h }}$ Lund University Hospital, Lund, Sweden; Department of Cardiology, ${ }^{\mathrm{i}}$ Kuopio University Hospital, Kuopio, Finland; Heart Center, ${ }^{\mathrm{j}}$ Tampere University Hospital, Tampere, Finland; and Department of Cardiothoracic Surgery, ${ }^{\mathrm{k}}$ St Olav's University Hospital and Institute for Circulation and Medical Imaging, University of Science and Technology, Trondheim, Norway.
}

patients. ${ }^{1}$ However, they are of limited durability owing to late degenerative valve failure and dense calcification that, at times, results in the need for reoperation. ${ }^{2-4}$ Transcatheter valve-in-valve (VinV) implantation is a new 


$$
\begin{aligned}
& \text { Abbreviations and Acronyms } \\
& \text { ID }=\text { internal diameter } \\
& \text { TAVI }=\text { transcatheter aortic valve implantation } \\
& \text { TEE }=\text { transesophageal echocardiography } \\
& \text { THV }=\text { transcatheter heart valve } \\
& \text { VinV }=\text { valve-in-valve }
\end{aligned}
$$

minimally invasive procedure that can offer an alternative to reoperative open surgery. Several reports have suggested that, in selected patients, the off-label use of transcatheter VinV replacement is feasible, with acceptable early results. ${ }^{5-10}$ The experience is, however, still limited, particularly with regard to the safety of implantation of the transcatheter heart valve (THV) into different types of failed bioprostheses, the hemodynamic performance, and longer term outcomes. In addition, comparative data are needed in terms of the feasibility, safety, and performance of the different THV devices.

The Nordic Valve-in-Valve registry was initiated with the objectives to further explore the utility of the VinV concept for the aforementioned issues. In addition, it was designed to capture all VinV-transcatheter aortic valve implantation (TAVI) procedures performed in a defined geographic area and thereby provide a real-world look at the incidence and prevalence of these procedures.

\section{METHODS \\ Registry Design}

The Nordic Valve-in-Valve Registry was initiated in December 2011. It is an independent, noncommercial physician-founded registry that does not receive any external funding. The invitation to participate in the registry was sent to Nordic centers with an active TAVI program, all of whom responded. The 11 centers that had performed at least 1 VinV-TAVI procedure were all included and contributed their comprehensive experience to the registry.

The data collection was retrospective using a dedicated case report form. The collected variables included detailed information of the make and type of the primary bioprosthesis and their dimensions, which were obtained from published charts. ${ }^{11}$ The pertinent demographics and patient risk factors were defined according to the criteria of the EuroSCORE (ES) registry. ${ }^{12}$ The baseline hemodynamics of the failed bioprosthesis was determined by the last transthoracic echocardiographic study obtained before the procedure. The mechanism of failure was classified as either stenosis or regurgitation, according to the America Society of Echocardiography criteria, and deemed to be combined when both stenosis and regurgitation were at least of moderate degree. The intraprocedural hemodynamic assessment of the degree of postimplantation aortic regurgitation was determined from transesophageal echocardiography (TEE) or transthoracic echocardiography (in cases performed with local anesthesia) and/or angiographic assessment. The periprocedural outcome and major postoperative clinical endpoints were assessed according to the Valve Academic Research Consortium criteria, apart from defining procedural success. ${ }^{13}$ This was defined as successful delivery and deployment with 1 transcatheter heart valve, VinV performance with neither severe stenosis (peak gradient $>60 \mathrm{~mm} \mathrm{Hg}$ or peak velocity $>4 \mathrm{~m} / \mathrm{s}$ ) nor moderate to severe regurgitation, no conversion to open heart surgery, and the patient leaving the operating theater or catheterization suite alive. The patients underwent postoperative clinical and echocardiographic follow-up according to the institutional protocols. High postprocedural gradients were defined according to the Valve Academic Research Consortium criteria as those with a mean transvalvular gradients of $20 \mathrm{~mm} \mathrm{Hg}$ or more.

The ethics committee of the Helsinki University Hospital (Helsinki, Finland) approved the study protocol.

\section{Patient Characteristics}

The study population consisted of 45 patients who had undergone aortic VinV-TAVI in 11 cardiac surgical centers in the Nordic countries from May 2008 to January 2012. Of the 45 patients, 26 were men (57.8\%), and the mean patient age was 80.6 years (range, 61-91). The baseline patient characteristics, including types and sizes of the failed bioprosthesis are listed in Tables 1 and 2. The mode of failure was classified as valve stenosis or regurgitation, or both, in $51 \%, 29 \%$, and $18 \%$ of the patients, respectively. The peak and mean preoperative gradient was $76.7 \pm 16.5 \mathrm{~mm} \mathrm{Hg}$ and 44.8 $\pm 11.1 \mathrm{~mm} \mathrm{Hg}$, respectively, and the valve opening area was $0.59 \pm 0.14$ $\mathrm{cm}^{2}$ (patients with aortic regurgitation were excluded). The mean logistic ES, ES II, and Society of Thoracic Surgeons (STS) score was $35.4 \pm$ $16.1,16.3 \pm 8.8$, and $15.0 \pm 10.8$, respectively. Of the 45 patients, 33 underwent the procedure with the Edwards SAPIEN/XT device (Edwards LifeSciences, Irvine, Calif) and 12 with the CoreValve device (Medtronic Inc, Minneapolis, Minn). The patients did not differ in their baseline characteristics between device groups, apart from more patients being preoperatively in New York Heart Association class IV in the SAPIEN group (42\% vs $18 \%, P=.032)$.

\section{Statistical Analysis}

Qualitative data are expressed as frequencies and percentages, and differences between the device groups were compared using the chi-square test or Fisher's exact test, as appropriate. Normally distributed quantitative data are expressed as the mean \pm standard deviation and skewed data as the median and interquartile range (first quartile minus third quartile). Continuous variables and proportions were compared using the Mann-Whitney nonparametric $U$ test or Student's $t$ test, as appropriate. Late survival was assessed using Kaplan-Meier analysis. Differences with a 2-sided $P<.05$ were considered statistically significant. The SPSS, version 17.0 (IBM, Armonk, NY), was used for the statistical calculations.

\section{RESULTS \\ Procedural Characteristics and Results}

Table 3 lists the procedural data and immediate outcomes. The device sizes used were $23 \mathrm{~mm}$ in $76 \%$ of the SAPIEN and $26 \mathrm{~mm}$ in all CoreValve VinV implantations (Table 3). For the SAPIEN, the transapical access route was the most predominant ( $76 \%$ ). In contrast, all but 1 CoreValve was implanted transfemorally. General anesthesia and TEE monitoring were used for most SAPIEN procedures but less frequently for CoreValve $(94 \%$ vs $42 \%$, $P=.001$; and $94 \%$ vs $33 \%, P=.001$, respectively). Preimplantation valvuloplasty was performed in $33 \%$ of the procedures.

No procedural mortality occurred. The technical success rate was $96 \%$ ( 43 of 45). One patient with a CoreValve device in a 23-mm Mitroflow (Sorin Group, Vancouver, British Columbia, Canada) prosthesis underwent a second valve implantation owing to a malposition of the first implanted CoreValve. The other technical failure resulted 
TABLE 1. Demographics and preoperative data

\begin{tabular}{|c|c|c|c|c|}
\hline Variable & All patients $(n=45)$ & SAPIEN $(\mathbf{n}=\mathbf{3 3})$ & CoreValve $(n=12)$ & $P$ value \\
\hline Gender & & & & .467 \\
\hline Male & $26(58)$ & $18(55)$ & $8(67)$ & \\
\hline Female & $19(42)$ & $15(45)$ & $4(33)$ & \\
\hline Age (y) & $80.6(61-91)$ & $80.0 \pm 5.6$ & $79.7 \pm 8.4$ & .187 \\
\hline Interval from sAVR (y) & $8.7 \pm 3.5$ & $8.9 \pm 3.7$ & $8.0 \pm 2.9$ & .214 \\
\hline sAVR type & & & & .210 \\
\hline Stented pericardial & $21(47)$ & $17(52)$ & $4(33)$ & \\
\hline Stented porcine & $16(28)$ & $12(36)$ & $4(33)$ & \\
\hline Stentless porcine & $5(11)$ & $3(9)$ & $2(17)$ & \\
\hline Homograft & $3(7)$ & $1(3)$ & $2(17)$ & \\
\hline $\mathrm{ID}^{*}(\mathrm{~mm})$ & & & & .600 \\
\hline$\leq 19$ & $10(23)$ & $8(24)$ & $2(20)$ & \\
\hline $19-21$ & $20(47)$ & $14(42)$ & $6(60)$ & \\
\hline$\geq 21$ & $13(30)$ & $11(33)$ & $2(20)$ & \\
\hline$\overline{\text { Unknown } \dagger}$ & 2 & - & 2 & \\
\hline Failure mode & & & & .140 \\
\hline Stenosis & $23(51)$ & $16(48)$ & $7(58)$ & \\
\hline Regurgitation & $13(29)$ & $8(24)$ & $5(42)$ & \\
\hline Combined & $8(18)$ & $8(24)$ & - & \\
\hline Unknown & $1(2)$ & $1(3)$ & - & \\
\hline \multicolumn{5}{|l|}{ EuroSCORE } \\
\hline Logistic & $35.4 \pm 16.1$ & $37.3 \pm 16.6$ & $28.4 \pm 12.5$ & .263 \\
\hline II & $16.3 \pm 8.8$ & $17.4 \pm 9.4$ & $12.7 \pm 5.5$ & .153 \\
\hline STS score & $15.0 \pm 10.8$ & $15.1 \pm 10.6$ & $14.8 \pm 5.5$ & .387 \\
\hline NYHA class & & & & $.032 \ddagger$ \\
\hline I & - & - & - & \\
\hline II & - & - & - & \\
\hline III & $30(67)$ & $19(58)$ & $11(92)$ & \\
\hline IV & $15(33)$ & $14(42)$ & $1(8)$ & \\
\hline $\operatorname{LVEF}(\%)$ & $46.3 \pm 12.8$ & $45.5 \pm 13.1$ & $48.3 \pm 12.7$ & .761 \\
\hline LVEF $<40 \%$ & $14(31)$ & $11(34)$ & $3(25)$ & .552 \\
\hline Mitral valve regurgitation & & & & .523 \\
\hline None or trace & $11(24)$ & $7(21)$ & $4(33)$ & \\
\hline Mild & $26(58)$ & $20(61)$ & $6(50)$ & \\
\hline Moderate & $5(11)$ & $3(9)$ & $2(17)$ & \\
\hline Moderate to severe & $3(7)$ & $3(9)$ & $0(0)$ & \\
\hline Previous MI & $6(13)$ & $6(19)$ & $0(0)$ & .107 \\
\hline CVD & $8(18)$ & $6(18)$ & $2(17)$ & .906 \\
\hline Diabetes & $8(18)$ & $4(12)$ & $4(33)$ & .100 \\
\hline Chronic renal disease & $9(20)$ & $6(18)$ & $3(25)$ & .532 \\
\hline Peripheral atherosclerosis & $9(20)$ & $8(24)$ & $1(8)$ & .331 \\
\hline COPD & $9(20)$ & $5(16)$ & $4(33)$ & .214 \\
\hline$>1$ Open heart surgery & $5(11)$ & $1(3)$ & $4(13)$ & $.004 \ddagger$ \\
\hline
\end{tabular}

from severe intravalvular aortic regurgitation caused by leaflet dysfunction of a SAPIEN device in a patient with 21-mm Freestyle prosthesis (Medtronic). That patient underwent a successful open surgical correction 3 days later. Two patients developed periprocedural myocardial infarction. Major vascular complications consisted of an occlusive external iliac artery dissection in 1 patient that was managed by endovascular stent grafting. At the end of the procedure, none of the patients had more than mild intravalvular aortic insufficiency, with the exception of the previously mentioned patient with leaflet dysfunction. One patient had a moderate paravalvular leak. A greater number of patients with the SAPIEN device had no or only trace paravalvular leaks $(82 \%$ vs $33 \%, P=.005)$. 
TABLE 2. Tissue valves used in the primary surgery

\begin{tabular}{llc}
\hline \multicolumn{1}{c}{ Valve type } & \multicolumn{1}{c}{ Trade name } & Patients (n) \\
\hline Stented porcine & Biocor/Epic & $3(7)$ \\
& Carpentier Edwards SAV & $10(22)$ \\
& Mosaic & $2(4)$ \\
& Mosaic Ultra & $1(2)$ \\
Stented pericardial & Shelhigh & $1(2)$ \\
& Mitroflow & $15(33)$ \\
& Magna & $1(2)$ \\
Stentless porcine & Perimount & $4(9)$ \\
& Soprano & $1(2)$ \\
Homograft & Medtronic Freestyle & $3(7)$ \\
& Toronto SPV & $1(2)$ \\
& & $3(7)$ \\
\hline
\end{tabular}

Data in parentheses are percentages. $S A V$, Supra-annular valve; $S P V$, stentless porcine valve.

\section{Thirty-Day Outcomes}

The mean in-hospital and intensive care unit stay was 8.0 days (range, 2-22) and 1.2 days (range, $0-4$ ), respectively. The 30 -day mortality was $4.4 \%$. One patient who had experienced a periprocedural myocardial infarction died on the second postoperative day. She had had a 23-mm SAPIEN device implanted by way of the transfemoral route in a 23$\mathrm{mm}$ Freestyle prosthesis. In the autopsy, the left coronary ostium was found to be partially obstructed by the torn porcine leaflet of the bioprosthesis. The other patient died of aspiration pneumonia and respiratory insufficiency. One patient had a major stroke. A permanent pacemaker was implanted in $7 \%$ of patients ( 1 patient with stented and 2 with stentless valves; $3 \%$ SAPIEN vs $17 \%$ CoreValve, $P=\mathrm{NS}$ ).

The peak and mean transvalvular gradient at 30 days had decreased to $28.9 \pm 14.9 \mathrm{~mm} \mathrm{Hg}$ and $16.4 \pm 8.7 \mathrm{~mm} \mathrm{Hg}$, respectively (Figure 1). These values did not differ between the SAPIEN and CoreValve groups. A total of $17 \%$ of patients (16\% of SAPIEN and $18 \%$ of CoreValve) had a mean valve gradient greater than $20 \mathrm{~mm} \mathrm{Hg}$, but included no patients with aortic insufficiency as the primary failure mode. At 30 days, $95 \%$ of patients were at New York Heart Association class I or II.

The internal diameter (ID) of the failed bioprostheses, as given by the manufacturer, was, on average, $2 \mathrm{~mm}$ smaller for the pericardial valves than for the porcine valves (19.7 \pm $0.43 \mathrm{~mm}$ pericardial vs $21.7 \pm 0.31 \mathrm{~mm}$ porcine, $P=.039$ ). This did not, however, translate to any differences in the 30day hemodynamic performance, because the respective peak and mean valve gradient for the pericardial and porcine valves was $32.3 \pm 14.1 \mathrm{~mm} \mathrm{Hg}$ vs $29.2 \pm 13.1$ $\mathrm{mm} \mathrm{Hg}(P=.708)$ and $17.5 \pm 8.2 \mathrm{~mm} \mathrm{Hg}$ vs $17.0 \pm 8.0$ $\mathrm{mm} \mathrm{Hg}(P=.695)$.

\section{Follow-up Data}

The mean follow-up period was 14.4 months (range, 0-43). The cumulative survival at 6,12 , and 24 months was $93.2 \%$, $88.1 \%$, and $84.1 \%$, respectively (Figure 2). Seven late deaths have occurred, of which 2 have been cardiac-related (prosthetic valve endocarditis at 3 months and congestive heart failure at 22 months) and 3 have been noncardiacrelated (subdural hemorrhage at 8 months, chronic myeloid leukemia at 8 months, and traumatic head injury at 14 months). The cause of death was unknown for 2 patients.

At 1 year, both the functional and hemodynamic followup status was available for 29 patients. Of these 29 patients, $89.7 \%$ remained in New York Heart Association functional class I or II. The peak and mean valve gradient was $26.8 \pm$ $11.2 \mathrm{~mm} \mathrm{Hg}$ and $15.3 \pm 7.8 \mathrm{~mm} \mathrm{Hg}$, respectively. A mean valve gradient of $20 \mathrm{~mm} \mathrm{Hg}$ or greater was measured in $22 \%$ of patients, with no correlation to the complication rates or overall survival.

\section{DISCUSSION}

In the Nordic countries, the current annual overall volume of TAVIs has been estimated to be 850 to 900 cases. Currently, the VinV-TAVI procedures constitute only $2 \%$ to $3 \%$ of the total case volume. This can be expected to grow with increasing experience in TAVI and because of a general trend toward lowering the age for the use of a bioprosthesis. ${ }^{1}$ Nevertheless, with few exceptions, the number of VinV-TAVI implantations for each individual center will likely remain low. In our report, the caseload averaged 4.5 patients per unit. This was in line with that reported by Dvir et $a l,{ }^{14}$ in which the case series of 202 patients with VinV implantations was derived from a total of 38 centers.

Just as with all low-volume procedures, the scientific evidence can be expected be based on multicenter observational clinical series. Studies with direct comparisons to the open reoperative aortic valve replacement alternative are unlikely to be performed. The reported mortality for an elective redo aortic valve surgery has showed a high variation from $2 \%$ to $30 \%$, which has correlated mostly with patient-associated risk factors. ${ }^{2,3}$ Reoperations have been associated with increased morbidity, longer hospital stays, and prolonged recovery. VinV-TAVI is a very attractive, less-invasive, alternative for many of these, particularly high-risk, patients. The patient selection for these alternatives needs to be weighed carefully and requires an indepth understanding of their potential and limits.

\section{Procedural Safety and Efficacy}

Our results are in accordance with those from published single- and multicenter experience demonstrating that VinV-TAVI is technically feasible and has a safety profile at least as good as TAVI in the treatment of native aortic valve stenosis. Because of the high-risk patient group (with an average logistic ES of $35.4 \%$ ) that was treated, the observed $4.4 \%$ overall mortality was very acceptable. In addition, the incidence of major complications was relatively low. This is in keeping with the first multicenter experience using the SAPIEN valve reported by Webb et al. ${ }^{6}$ Their report of 24 
TABLE 3. Perioperative data and immediate outcomes

\begin{tabular}{|c|c|c|c|c|}
\hline Variable & All patients $(n=45)$ & SAPIEN $(n=33)$ & CoreValve $(n=12)$ & $P$ value \\
\hline \multicolumn{5}{|l|}{ Device size } \\
\hline 23 & $25(56)$ & $25(76)$ & - & NA \\
\hline 26 & $19(42)$ & $7(21.2)$ & 12 & \\
\hline 29 & $1(2)$ & $1(3)$ & - & \\
\hline Access route & & & & NA \\
\hline Transapical & $25(56)$ & $25(76)$ & - & \\
\hline Transfemoral & $17(38)$ & $6(18)$ & $11(92)$ & \\
\hline Transaortic & $2(4)$ & $2(6.1)$ & - & \\
\hline Subclavian & $1(2)$ & - & $1(8)$ & \\
\hline General anesthesia & $36(80)$ & $31(94)$ & $5(42)$ & $.001^{*}$ \\
\hline TEE & $35(78)$ & $31(94)$ & $4(33)$ & $.001^{*}$ \\
\hline Predilation valvuloplasty & $15(33)$ & $11(33)$ & $4(33)$ & .678 \\
\hline \multicolumn{5}{|l|}{ Procedural outcome } \\
\hline Mortality & - & - & - & \\
\hline Technical success & $43(96)$ & $32(97)$ & $11(92)$ & .208 \\
\hline Second valve implanted & $1(2)$ & - & $1(8)$ & NA \\
\hline \multicolumn{5}{|l|}{ Intravalvular AI } \\
\hline None or trace & $42(93)$ & $30(91)$ & $12(100)$ & \\
\hline Mild & $2(4)$ & $2(6)$ & - & \\
\hline Moderate & - & - & - & \\
\hline Severe & $1(2)$ & $1(2)$ & - & .557 \\
\hline \multicolumn{5}{|l|}{ Paravalvular AI } \\
\hline None or trace & $31(69)$ & $27(82)$ & $4(33)$ & \\
\hline Mild & $13(29)$ & $6(18)$ & $7(58)$ & \\
\hline Moderate & $1(2)$ & - & $1(8)$ & $.005^{*}$ \\
\hline Conversion to open surgery & $1(2)$ & $1(3)$ & - & NA \\
\hline Major vascular complication & $1(2)$ & - & $1(8)$ & NA \\
\hline Bleeding & & & & 692 \\
\hline Major & - & - & - & \\
\hline Minor & $6(13)$ & $4(12)$ & $2(17)$ & \\
\hline \multicolumn{5}{|l|}{ Outcome at $30 \mathrm{~d}$} \\
\hline Mortality & $2(4)$ & $2(6)$ & - & NA \\
\hline ICU stay $(d)$ & $1.2 \pm 0.7$ & $1.3 \pm 0.8$ & $0.9 \pm 0.6$ & .318 \\
\hline Hospital stay (d) & $8.0 \pm 3.9$ & $7.8 \pm 4.3$ & $8.4 \pm 2.9$ & .600 \\
\hline Periprocedural MI & $2(4)$ & $2(6)$ & - & NA \\
\hline Major stroke & $1(2)$ & $1(3)$ & - & NA \\
\hline Permanent pacemaker & $3(7)$ & $1(3)$ & $2(17)$ & .150 \\
\hline Paravalvular AI & & & & $.001 *$ \\
\hline None or trace & $34(79)$ & $29(94)$ & $5(42)$ & \\
\hline Mild & $8(19)$ & $2(6)$ & $6(50)$ & \\
\hline Moderate & $1(2)$ & - & $1(8)$ & \\
\hline Peak valve gradient $(\mathrm{mm} \mathrm{Hg}$ ) & $28.9 \pm 14.9$ & $28.7 \pm 10.6$ & $29.2 \pm 20.9$ & .070 \\
\hline Mean valve gradient $(\mathrm{mm} \mathrm{Hg})$ & $16.4 \pm 8.7$ & $16.2 \pm 6.6$ & $16.7 \pm 12.4$ & .071 \\
\hline Valve area $\left(\mathrm{cm}^{2}\right)$ & $1.3 \pm 0.4$ & $1.2 \pm 0.3$ & $1.4 \pm 0.5$ & .065 \\
\hline LVEF & $49.6 \pm 11.7$ & $51.1 \pm 10.7$ & $46.6 \pm 13.7$ & .398 \\
\hline \multicolumn{5}{|l|}{ NYHA functional class } \\
\hline I & $16(37)$ & $11(35)$ & $5(42)$ & \\
\hline II & $22(51)$ & $15(48)$ & $7(52)$ & \\
\hline III & $1(2)$ & $1(3)$ & - & \\
\hline IV & - & - & - & \\
\hline Unknown & $4(9)$ & $4(13)$ & - & .796 \\
\hline
\end{tabular}

Data presented as number of patients ( $\%)$, mean \pm standard deviation, or median (interquartile range). NA, Not available; TEE, transesophageal echocardiography; AI, aortic insufficiency; $M I$, myocardial infarction; ICU, intensive care unit; NYHA, New York Heart Association; $L V E F$, left ventricular ejection fraction. *Statistically significant. 

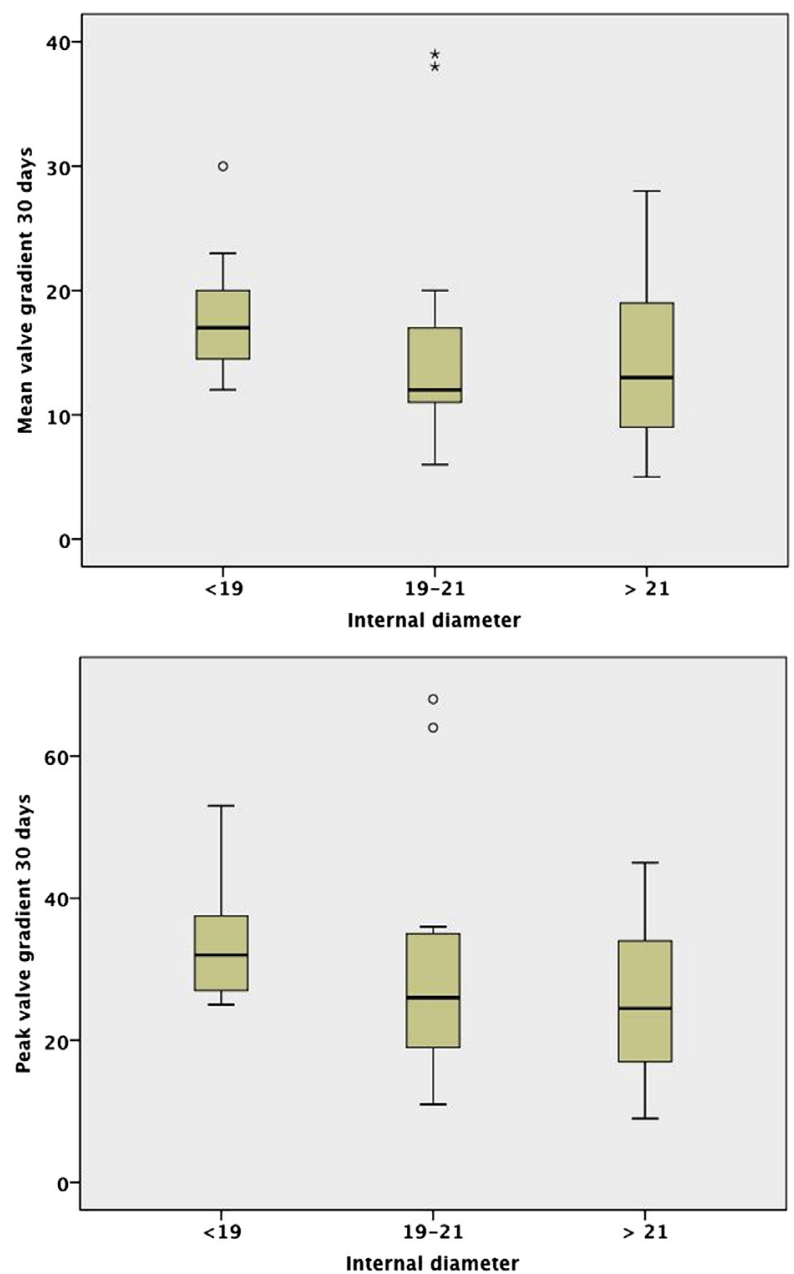

FIGURE 1. The mean and peak valve gradient 30 days after valve in valve transcatheter aortic valve implantation for different manufacturer-given internal diameters (IDs) was $18.3 \pm 6.2 \mathrm{~mm} \mathrm{Hg}, 16.4 \pm 10.0 \mathrm{~mm} \mathrm{Hg}$, and $14.4 \pm 7.5 \mathrm{~mm} \mathrm{Hg}$ and $34.14 \pm 9.9 \mathrm{~mm} \mathrm{Hg}, 30.3 \pm 16.6 \mathrm{~mm} \mathrm{Hg}$, and $25.6 \pm 12.3 \mathrm{~mm} \mathrm{Hg}$ for an ID of less than $19 \mathrm{~mm}, 19$ to $21 \mathrm{~mm}$, and greater than $21 \mathrm{~mm}$, respectively.

patients resulted in a 30-day mortality of $4.2 \%$ of patients with a mean logistic ES of $30.4 \%$. Similar results were achieved in a single-center VinV experience with the SAPIEN valve for 23 high-risk patients, with no operative mortality. ${ }^{15}$ In the largest multicenter experience of VinV-TAVI, most implantations were performed using the CoreValve device. $^{14}$ The 30 -day mortality was $8.4 \%$ in a patient cohort with a risk profile similar to that in the other cited studies.

However, concerns exist of major procedure-specific safety hazards with VinV-TAVI. These include device malposition, an increased risk of coronary obstruction, especially with certain valve types, and suboptimal systolic valve function.

The placement of the device inside the bioprosthesis must be very accurate, because the area of anchoring is very narrow and, in the case of a stented bioprosthesis, limits itself to a circular basal ring. This dictates how much and which

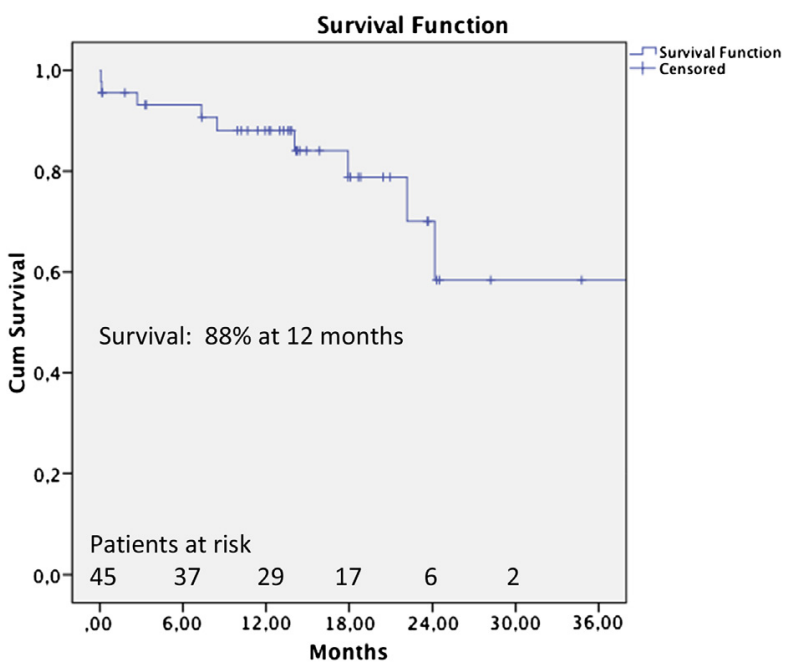

FIGURE 2. The overall postoperative survival of the 45 valve in valve transcatheter aortic valve implantation patients.

parts of the THV can expand. To facilitate VinV-TAVI, the early reports preferred the use of the direct surgical access (typically transapical) route for better device control and more accurate placement. ${ }^{6}$ With the device modifications, the transfemoral access route has become a widely accepted alternative. However, a rate of up to $15.3 \%$ of initial device malposition has been reported. ${ }^{14}$ In our results, only 1 patient required a second valve implantation because of a malpositioned first device. It is a matter of debate whether this result is a reflection of more than $60 \%$ use of shorter surgical access routes.

An increased risk of coronary obstruction has been associated with certain valve types, including stented pericardial valves with a design that has the pericardial leaflet wrapped outside of the stent frame and stentless valves. Our report included 15 VinV-TAVI with the stented pericardial valve described (Mitroflow), with no coronary obstruction observed. That compares favorably with the previously reported coronary obstruction incidence of $7.7 \%$ in VinV implantations inside the Mitroflow prosthesis. Nevertheless, patients with these valve types should be studied very carefully, especially in cases of small aortic root dimensions and a high supra-annular position of the bioprosthesis. Computed tomographic angiography with multiplanar reconstructions is likely the best assessment method.

The stentless valves, including homografts, pose unique challenges for VinV positioning and deployment because of their size, lack of radiopaque markers, heterogeneous design, and implantation techniques. These issues were reported by Bapat et al. ${ }^{15} \mathrm{We}$ suggest that more oversizing $(>10 \%$ in the cross-sectional area) is needed because failed stentless valves have typically not been very calcified and do not have a frame in which to anchor. Hence, self-expanding devices that allow for more oversizing but exert less radial force have been preferred by some investigators in these 
situations. ${ }^{16}$ In the presence of severe aortic regurgitation, the aortic annulus might not be easily delineated. Special measures could be necessary to create landmarks for secure valve positioning, such as placing a pigtail catheter in the base of the sinus or marking the left main coronary ostium with a guidewire. The risk of complications, even when taking all precautions, is still most likely greater than with the stented valves. In our series, with 5 stentless porcine valves, 2 patients experienced major complications, coronary obstruction in 1 patient and leaflet dysfunction requiring conversion to an open procedure in 1 patient. The 3 homograft cases were performed without complications.

\section{Valve Sizing and Hemodynamic Function of VinV-TAVI}

Choosing the optimal THV size for the failed bioprosthesis is critical for secure fixation and optimal hemodynamic function. Valve embolization in VinV-TAVI has been reported. Paravalvular aortic regurgitation seems to have been less of a problem in the published series of VinVTAVI compared with TAVI of native aortic valve stenosis. However, excessive oversizing can lead to incomplete expansion of the new valve. This in turn could cause valve leaflet distortion, affecting systolic hemodynamic function and potentially limiting the durability of the THV. This was demonstrated in an in vitro model evaluating the hemodynamic performance of a $23-\mathrm{mm}$ THV.${ }^{17}$ It was shown that, when implanted in 19- and 21-mm bioprostheses, the incomplete stent expansion resulted in leaflet distortion and central regurgitation. In clinical series, the valve gradients after VinV-TAVI have been significantly greater than those after TAVI in the native aortic valve, in which a mean gradient of less than $10 \mathrm{~mm} \mathrm{Hg}$ typically can be expected. ${ }^{18}$

The size of the THV used should match or exceed the ID of the bioprosthesis. The labeled IDs can be obtained from published charts. ${ }^{11}$ However, the exact or "true" ID might be different from the manufacturer-given ID. ${ }^{19}$ In the case of stented valves, the ID is invariably smaller by 1 to 3 $\mathrm{mm}$. Owing to their design, the stented porcine valve prostheses typically represent the upper range. This was also shown in the present study, in which the VinVTAVIs on porcine valves had, on average, 2-mm larger manufacturer-given IDs than the pericardial valves, resulting in similar postprocedural valve gradients. In addition, the actual ID can be decreased further by various disease processes, such as calcification, fibrosis, and pannus formation. A thorough preprocedural screening with computed tomography and TEE is therefore necessary to identify any discrepancies between the nominal diameters and the in vivo measurements. However, little is known regarding the TEE and computed tomography measurements and their correlation to the actual ID.

It has been postulated that THVs with a "supra-annular" design (eg, CoreValve) could perform hemodynamically better in small valve sizes than an "intra-annular" device (eg, SAPIEN). This was supported by Dvir et al, ${ }^{14}$ who found the SAPIEN device to have greater valve gradients for manufacturer-given IDs less than $20 \mathrm{~mm}$ than did the CoreValve device. In our experience, we could verify, in accordance with their results, that close to $20 \%$ of patients will have a mean transvalvular gradient exceeding $20 \mathrm{~mm}$ $\mathrm{Hg}$ but could not demonstrate any difference between the devices.

\section{Role of Balloon Aortic Valvuloplasty}

Only a few patients underwent balloon aortic valvuloplasty, with seemingly no major procedural hazards in terms of difficulties when crossing the valve or challenges in the hemodynamic treatment of these patients. In conjunction with VinV-TAVI, the balloon aortic valvuloplasty can most likely be reserved for selected patients such as those with very critical aortic stenosis or with an identified increased risk of coronary obstruction; performing a root angiogram with the balloon inflated can be a useful method of assessing the risk.

\section{Implications for Surgical Aortic Valve Replacement Practice}

The increasing evidence of an acceptable safety profile of VinV-TAVI will most likely accelerate the trend of choosing a biologic valve prosthesis instead of a mechanical valve even for younger patients undergoing primary surgical aortic valve replacement. For patients who have a high likelihood of outliving the estimated durability of their bioprosthesis, the surgical aortic valve replacement should be performed in such a manner that good prerequisites for a successful future VinV-TAVI exist. The valve size should be large enough to minimize residual valve gradients and the risk of primary patient-prosthesis mismatch. This might change the practice toward performing more aortic root dilation annuloplasty procedures to accommodate a large enough valve size. In addition, the chosen primary valve prosthesis should preferably be of a type not known to have an increased risk of procedural complications at VinV-TAVI, most importantly, a low risk of coronary obstruction.

\section{Study Limitations}

The present study had several limitations with respect to both its design and patient material. As a registry trial, the data were not audited, and the collected hemodynamic data had not been core laboratory adjudicated. The number of patients was still relatively small, precluding additional analysis of the safety profile of VinV-TAVI in different primary valve types and comparing the performance of the available THV devices. The results can be regarded as hypothesis-generating only. The mean follow-up was limited to little more than 1 year. Thus, the long-term 
sequelae of VinV-TAVI, especially with regard to the effect of high postprocedural valve gradients or durability, could not be assessed.

\section{CONCLUSIONS}

VinV-TAVI is a part of the armamentarium in most centers performing TAVI, albeit in small numbers. In this highrisk patient population, the early experience has been excellent, with a low incidence of device- or procedure-related complications. However, a considerable number of patients had a high transvalvular gradient after the procedure, the long-term effects of which are unknown. Close long-term surveillance after VinV-TAVI is needed, especially for patients known to have suboptimal systolic valve function. Finally, future larger scale studies are warranted.

The authors thank Janne Jokinen from Helsinki University Hospital (Helsinki, Finland) for his invaluable aid and review of the statistical analysis.

\section{References}

1. Brown JM, O'Brien SM, Wu C, Sikora JA, Griffith BP, Gammie JS. Isolated aortic valve replacement in North America comprising 108,687 patients in 10 years: changes in risks, valve types, and outcomes in the Society of Thoracic Surgeons National Database. J Thorac Cardiovasc Surg. 2009;137:82-90.

2. Maganti M, Rao V, Armstrong S, Feindel CM, Scully HE, David TE. Redo valvular surgery in elderly patients. Ann Thorac Surg. 2009;87:521-5.

3. Balsam LB, Grossi EA, Greenhouse DG, Ursomanno P, Deanda A, Ribakove GH, et al. Reoperative valve surgery in the elderly: predictors of risk and long-term survival. Ann Thorac Surg. 2010;90:1195-200; discussion 1201.

4. Jones JM, O'Kane H, Gladstone DJ, Sarsam MA, Campalani G, MacGowan SW, et al. Repeat heart valve surgery: risk factors for operative mortality. $J$ Thorac Cardiovasc Surg. 2001;122:913-8.

5. Wenaweser P, Buellesfeld L, Gerckens U, Grube E. Percutaneous aortic valve replacement for severe aortic regurgitation in degenerated bioprosthesis: the first valve in valve procedure using the CoreValve revalving system. Catheter Cardiovasc Interv. 2007;70:760-4.

6. Webb JG, Wood DA, Ye J, Gurvitch R, Masson JB, Rodes-Cabau J, et al. Transcatheter valve-in-valve implantation for failed bioprosthetic heart valves. Circulation. 2010;121:1848-57.

7. Khawaja MZ, Haworth P, Ghuran A, Lee L, de Belder A, Hutchinson N, et al. Transcatheter aortic valve implantation for stenosed and regurgitant aortic valve bioprostheses: CoreValve for failed bioprosthetic aortic valve replacements. J Am Coll Cardiol. 2010;55:97-101.

8. Kempfert J, Van Linden A, Linke A, Borger MA, Rastan A, Mukherjee C, et al. Transapical off-pump valve-in-valve implantation in patients with degenerated aortic xenografts. Ann Thorac Surg. 2010;89:1934-41.

9. Attias D, Himbert D, Hvass U, Vahanian A. "Valve-in-valve" implantation in a patient with stentless bioprosthesis and severe intraprosthetic aortic regurgitation. J Thorac Cardiovasc Surg. 2009;138:1020-2.

10. Ihlberg L, Sahlman A, Sinisalo J, Rapola J, Laine M. Transaortic valve-in-valve implantation after previous aortic root homograft. Ann Thorac Surg. 2012;94: 1718-21.

11. Bapat V, Mydin I, Chadalavada S, Tehrani H, Attia R, Thomas M. A guide to fluoroscopic identification and design of bioprosthetic heart valves: a reference for valve-in-valve procedure. Catheter Cardiovasc Interv. 2013;81:853-61.

12. Roques F, Nashef SA, Michel P, Gauducheau E, de Vincentiis C, Baudet E, et al. Risk factors and outcome in European cardiac surgery: analysis of the EuroSCORE multinational database of 19030 patients. Eur J Cardiothorac Surg. 1999;15:816-22; discussion 822-3.

13. Leon MB, Piazza N, Nikolsky E, Blackstone EH, Cutlip DE, Kappetein AP, et al. Standardized endpoint definitions for transcatheter aortic valve implantation clinical trials: a consensus report from the Valve Academic Research Consortium. J Am Coll Cardiol. 2011;57:253-69.
14. Dvir D, Webb J, Brecker S, Bleiziffer S, Hildick-Smith D, Colombo A, et al. Transcatheter aortic valve replacement for degenerative bioprosthetic surgical valves: results from the Global Valve-in-Valve Registry. Circulation. 2012; 126:2335-44.

15. Bapat V, Attia R, Redwood S, Hancock J, Wilson K, Young C, et al. Use of transcatheter heart valves for a valve-in-valve implantation in patients with degenerated aortic bioprosthesis: technical considerations and results. J Thorac Cardiovasc Surg. 2012;144:1372-80.

16. Piazza N, Bleiziffer S, Brockmann G, Hendrik R, Deutsch MA, Opitz A, et al. Transcatheter aortic valve implantation for failing surgical aortic bioprosthetic valve: from concept to clinical application and evaluation (part 2). JACC Cardiovasc Interv. 2011;4:733-42.

17. Azadani AN, Jaussaud N, Matthews PB, Ge L, Chuter TAM, Tsengl EE. Transcatheter aortic valves inadequately relieve stenosis in small degenerated bioprostheses. Interact Cardiovasc Thorac Surg. 2010;11:70-7.

18. Leon MB, Smith CR, Mack M, Miller DC, Moses MW, Svensson LG, et al. Transcatheter aortic-valve implantation for aortic stenosis in patients who cannot undergo surgery. N Engl J Med. 2010;363:1597-607.

19. Von Oppell UO, Segadal L, Busund R, Johnston GG, Dimitrakakis G, Masani N, et al. Aortic annulus diameter and valve design each determining the valve size implanted. J Heart Valve Dis. 2012;12:591-8.

\section{Discussion}

Dr Mathew R. Williams (New York, NY). This is an exciting procedure that really represents a sweet spot for transcatheter valves. This is an observational study, your data is well presented, and to that end I certainly do not have any critiques. However, I would rather spend the time to discuss procedural and planning components, because, fortunately now, a lot more of us are able to do this procedure.

The first question is how have you decided what kind of access to use in these patients? Initially, the reports suggested that this should be done with a transapical approach. We have not found that and have generally been just access driven.

Dr Ihlberg. I would say that the access is completely today up to the discretion and preference of the operator and also partly for the device used at the given center. My personal bias is that I still like the surgical short access, good device maneuvering, and to be able to land the device exactly on the target. I tend to conceptualize the procedure as being similar to landing a helicopter onto an air carrier. Needless to say, we do not have any air carriers in Finland, and I have never done that myself, but it is just a concept. Still, I like it, especially when you do valve in valve because of the sort of small target in which you land it, but I know that, equally well, people do this transfemorally.

Dr Williams. Is there any reason that this procedure works very well? There is much less paravalvular leakage than we see in the native aortic stenosis, which is really one of the Achilles heels of this procedure. Is there any reason that in most patients this should not quickly become the standard of care for degenerated tissue valves, even in lower risk patients?

Dr Ihlberg. That is a great question or thought, and I think you are correctly right in your considerations. I believe that things are rapidly moving to that direction, so that valve-in-valve TAVI might even become a default procedure under certain conditions. Thus, if you do not have any pre-identified risk of coronary obstruction or if you do not have a primary patient-prosthesis mismatch in these patients, you can expect to have a reasonable gradient after the procedure. The results so far in our study and in the other studies, of which the largest is the global valve-in-valve registry, have been good. 